\title{
Violencia doméstica, consumo de sustancias y otras circunstancias concurrentes. ¿El derecho versus derecho a la salud?
}

RESUMEN: Tras reflexionar sobre la violencia de género padecida sobre todo en el ámbito doméstico, se aportan algunos datos procedentes de un estudio realizado sobre las sentencias dictadas durante un año en el Paíís Vasco y referidas a tipología delictiva relacionada con la violencia intrafamiliar. Los resultados reflejan que el consumo de alcohol, de otros tipos de drogas, la existencia de trastornos mentales o la precariedad económica no aparecen como factores criminógenos tan determinantes como en otros trabajos anteriormente realizados sobre el tema. Las medidas y actuaciones en muy diversos campos de intervención son una urgente necesidad.

PALABRAS CLAVE: violencia, ámbito familiar, alcohol, otras drogas, pobreza, trastorno mental

\begin{abstract}
SUMMARY: After a reflection about gender and violence mainly in the family area, there are some data from a research into sentences of domestic violence in Basque Country during one year. The results show that alcohol, other drugs, mental diseases or poverty are not criminal factors as determinant as in other essays about the same topic. The measures and interventions in a large range of areas are now an urgent need.

KEY WORDS: domestic violence, family, alcohol, drugs, poverty mental disease.
\end{abstract}

\section{Introducción.-}

La violencia en el ámbito familiar no es un fenómeno reciente. El "síndrome del niño golpeado", la "mujer masoquista" o "la agresión a las mujeres" ya fueron descritos en los años sesenta por autores diversos. Sería una década más tarde cuando, por influjo del nuevo y floreciente movimiento feminista, fueron expresadas socialmente las formas y consecuencias de esta violencia (1), constatándose que los efectos de esas agresiones son múltiples en las víctimas directas: las mujeres, y los hijos e hijas. Así, la violencia de género tiene como víctimas principales a las mujeres de cualquier edad, raza, cultura, clase o condición social, aunque con mayor afectación en las capas sociales más desfavorecidas. Es, por tanto, una violencia de género trasversal. El maltrato a las mujeres no tiene ideología por más que sea una imposición de la cultura patriarcal y que ciertos regímenes políticos lo hayan fomentado, y todavía lo fomenten, mediante el abuso o la discriminación de la mujer. Por citar sólo un ejemplo y sin tener que irnos a países de cultura islámica o demasiado atrás en el tiempo, durante el régimen franquista las españolas casadas eran 
prácticamente consideradas como incapaces para la realización de determinados actos civiles. Hasta 1975 existió la ley “del cabeza de familia” según la cual una mujer no podía ni abrir una cartilla de ahorros a su nombre. El Código Civil en su art.52 estipulaba que la mujer debía obediencia al marido. Más cerca aún, hasta el año 1989, en la legislación penal ni siquiera estaba contemplado el maltrato psicológico.

La violencia contra las mujeres es definible como "todo acto de violencia por razón de género que produce o puede generar daño físico, sexual, psicológico o sufrimiento a las mujeres, incluidas las amenazas de tales actos, la coerción o las privaciones arbitrarias de la libertad, concurriendo esto tanto en el ámbito público como en el privado" (2) ${ }^{1}$. La violencia de género, ejercida en gran medida en el ámbito doméstico, por varones mayoritariamente, es un problema complejo y multifactorial en su etiología y repercusiones. Se basa en mecanismos de dominio de unas personas sobre otras, de hombres sometiendo a mujeres, pretendiendo señalar quien ostenta el poder en el marco familiar con formas violentas de interacción, como es el caso de las relaciones de abuso. Además, estas relaciones son resultado de un modelo familiar y social que las ha aceptado como estrategias váálidas para la resolución de ciertos conflictos. Parece que muchos hombres han perdido el control de sus familias $\mathrm{y}$, en muchas ocasiones, de sus trabajos, lo cual ha creado una percepción subjetiva de creciente impotencia, reaccionando de modo defensivo con su agresividad misógina y a veces autodestructiva (3). Por tanto, creemos que esta violencia es un problema "para" las mujeres y un problema "de" los hombres (4). Son generalmente hombres quienes ejercen comportamientos violentos y mujeres quienes los padecen. Son los actores de esta tragedia enmarcada en un entorno social y cultural que ha venido tolerando e incluso propiciando estas agresiones con el consenso ideológico que justifica la interiorización de roles victimistas por las mujeres, llegando incluso a anestesiar su malestar.

Las dificultades de expresión emocional, la débil autoestima, la percepción distorsionada de la realidad y la vivencia de amenaza consecuente, son la base de muchos conflictos violentos en el hogar. Por eso, la forma más frecuente de violencia contra las mujeres es la doméstica a través del maltrato repetido mediante abuso físico, sexual y/o psicológico. Empujones, golpes, violaciones, insultos, humillaciones, amenazas o incluso asesinatos no son sino algunas de las manifestaciones de estas agresiones en el ámbito doméstico que cotidianamente ocupan un lugar destacado en los medios de comunicación.

Según datos de la Comisión Europea las cifras son preocupantes: una de cada cinco mujeres de la Unión Europea ha sufrido malos tratos alguna vez en su vida. Una mujer de cada diez que vive en pareja ha sido víctima de violencia por su marido o compañero (5). Además, se ha constatado en la "Resolución de la Comisión de Derechos Humanos sobre la eliminación de la violencia contra la mujer" (1997/48), cómo algunos grupos de mujeres, como las que pertenecen a grupos minoritarios o las mujeres con discapacidad, son particularmente vulnerables a este tipo de violencia. 
En el Estado Español, en el año 2001, se produjeron 42 muertes relacionadas con la violencia doméstica, según datos proporcionados por el Ministerio de Asuntos Sociales $^{2}$.(6) Esta cifra sensiblemente menor a la de 2000, año en que fallecieron 72 mujeres por agresiones de sus maridos o compañeros, habría que matizarla teniendo en cuenta que estos datos se refieren únicamente a mujeres muertas a mano de su cónyuge o de su pareja de hecho. Es decir no se reflejan las muertes producidas por los ex-cónyuges, ex-parejas, novios, ex-novios, ascendientes, descendientes o hermanos. Si tuviéramos en cuenta también estas variables, la cifra de mujeres muertas sería bastante mayor (en este sentido, algunas publicaciones o asociaciones de mujeres duplican la cifra de mujeres fallecidas de forma violenta ${ }^{3}$. Además, hay que tener en cuenta que estas muertes se producen con frecuencia tras numerosas denuncias, intentos de separación, separaciones, etc. Son 2,5 millones de mujeres en contexto de pareja las que sufren la violencia según la macroencuesta del Instituto de la Mujer del año 2000. Mujeres agredidas por maltratadores que casi nunca padecen trastornos mentales como con demasiada frecuencia se argumenta.

No obstante, aunque pequeña, hay una relación entre violencia familiar y enfermedades mentales, pudiendo existir en algunas personas rasgos de personalidad acentuados -dependencia emocional, agresividad, celos, déficit de autoestima, etc- pero sin trastorno psicopatológico en la mayoría de los maltratadores (7). Se estima en solo un $4 \%$ quienes tienen algún trastorno diagnosticable según los criterios médicos al uso, siendo "normales" la inmensa mayoría desde el punto de vista patológico, por más que sus vínculos, modos de comunicación y comportamientos en el seno familiar o en el hogar sean intolerables.

Un 24\% de las mujeres con edades entre 18 y 64 años son víctimas de malos tratos. De ellas, un $12 \%$ han pensado alguna vez en el suicidio para dar fin a su situación. Incluso un $6 \%$ de las mujeres maltratadas ha intentado quitarse la vida en alguna ocasión ${ }^{4}$. La afectación psicopatológica es, asimismo, sufrida por las mujeres que reciben maltrato físico o psicológico (8), y un elevado número de ellas también padecen relaciones sexuales forzadas.

Además, en estos últimos años también se ha producido un aumento en el número de denuncias, hasta el punto que en el año 2000 fueron presentadas 22.367 denuncias en los juzgados del Estado español por motivos que pueden ser considerados como de violencia doméstica. Ha aumentado considerablemente la cantidad de mujeres que optan por denunciar los malos tratos. A pesar de ello, se considera que el $85 \%$ de las situaciones de violencia doméstica no llegan a denunciarse $(9,18)$. Sin embargo el problema no queda ahí, ya que pese al gran número de denuncias que se interponen la mayoría de ellas no llegan a la fase de diligencias previas, porque son retiradas por las mujeres ante presiones, sentimientos de culpa o miedos de todo tipo, o porque están mal formuladas en los dificultosos trámites administrativos. Además, la falta de apoyos 
legales o jurídicos y el desconocimiento de los recursos existentes (casas de acogida, pisos tutelados, asesoramiento psicológico, etc.) no ayudan a que las denuncias sigan su normal trámite.

Con todos estos datos se puede considerar que existe cierta falta de voluntad para castigar a quien ejerce violencia en el entorno intrafamiliar, lo que deriva en múúltiples problemas tanto jurídicos como, principalmente, sociales.

La mayoría de la personas agredidas por maltrato doméstico han tomado contacto con los dispositivos sanitarios del sistema de salud (10), sobre todo a través de los servicios de urgencia, de atención primaria, ginecología, gastroenterología y reumatología (11) donde son atendidas principalmente de lesiones de defensa, fracturas, roturas timpánicas, somatizaciones de diversa índole, abuso de sustancias, cuadros ansioso-depresivos o trastornos de estrés postraumático. Pero a pesar de que está asumido ampliamente que estas personas son más vulnerables a padecer algún trastorno sobre la salud mental, la violencia en el ámbito del hogar no ha sido objeto de interés para la comunidad científica psiquiátrica, salvo atenciones esporádicas por profesionales de la psicología y la psiquiatría. Comprensible si pensamos que la psiquiatría ha considerado el discurso de género como algo periférico y no fundamental en la organización del pensamiento (12). La violencia sexista es, ante todo, un fenómeno psicosocial con una amplia problemática que obliga a considerarla como cuestión de Derechos Humanos, y como tal debiera ser considerada desde los ámbitos institucionales, requiriendo cuestionar determinados prejuicios culturales y de género.

\section{Un estudio}

Para este artículo se han tomado algunos resultados parciales situados en el marco de una investigación sobre "La violencia en el ámbito doméstico y su tratamiento en el ámbito de la Administración de Justicia" en la provincia de Bizkaia, desarrollada en el año 2000 y referidos al año anterior. La totalidad de los casos de Bizkaia, unido a los datos muestrales obtenidos en Gipuzkoa y Araba, en virtud de otro estudio efectuado a nivel estatal, ha permitido una aproximación rigurosa al análisis de este fenómeno y su tratamiento en la Administración de justicia dentro de la Comunidad Autónoma Vasca.

Los datos proceden del trabajo de campo realizado en todas los partidos judiciales del País Vasco para este estudio encargado por el Consejo General del poder Judicial y desarrollado desde la Universidad de Zaragoza.

\section{Metodología}

Se extrajeron datos de los expedientes correspondientes a las sentencias dictadas durante un año -1999- en los casos referidos a una tipología delictiva relacionada con la violencia intrafamiliar. El estudio de las sentencias y procedimientos a realizar ha 
INFORMES

abarcado delitos contra la vida; delitos de lesiones; delitos contra la libertad sexual; delitos contra la inviolabilidad del domicilio; delitos contra los derechos y deberes familiares; faltas contra las personas.

Se partió de un concepto de violencia doméstica de naturaleza fundamentalmente subjetiva, que atienda a las personas que se ven implicadas como autores o como víctimas y no meramente al espacio físico del domicilio. La violencia doméstica, abarca en este sentido a aquellas personas entre las que existen vínculos parentales (padres e hijos, maridos y esposas, y las situaciones análogas) y ello incluso cuando dichos víínculos puedan estar en plena crisis o la violencia se presente ligada a ella. En este sentido, el objeto del estudio no se ha limitado a la violencia doméstica contra la mujer, aunque este es uno de los aspectos fundamentales, sino que se aborda desde una perspectiva global, prestando especial consideración al maltrato infantil y a su relación con las demás manifestaciones de violencia doméstica.

En la ficha técnica o protocolo para la recogida de datos se reflejaron items referentes a determinadas características de las personas afectadas por malos tratos y de sus infractores. A continuación en este artículo se analiza la información aportada por algunos de estos items, concretamente los referentes a determinadas circunstancias personales concurrentes en el agresor como son el alcoholismo, otro tipo de drogadicción, trastorno mental, desempleo o necesidades económicas. Todo ello, atendiendo a las diferentes personas que padecen la violencia: la pareja, los descendientes, ascendientes, hermanos, etc.

Para el análisis de resultados se utilizó el programa informático SPSS.

\section{Resultados}

Tras la recogida de estos datos y analizada la información se ofrece un perfil demográfico y psicopatológico de las personas agresoras en el ámbito doméstico, pudiendo observarse algunos resultados que vinculan la violencia familiar con el consumo de sustancias y los trastornos en la salud mental. Aunque es claramente minoritario este porcentaje, no por ello carece de significación.

En los expedientes de las personas con sentencia firme se reflejaba el consumo de alcohol como elemento alterador del comportamiento, en porcentajes que han oscilado entre el $11,7 \%$ y el $15,5 \%$, según fueran ascendientes, descendientes o sus parejas las personas afectadas (Tabla 1). Así mismo, se detectó un consumo de otro tipo de drogas ( en torno al 5\%) y trastornos mentales (alrededor del 4\%) cuando las agresiones eran padecidas por la pareja o por los hijos, mientras que estos porcentajes se elevaban hasta el $13-14 \%$ si los agredidos eran familiares ascendientes (hermanos,...) 
Tabla 1. CIRCUNSTANCIAS PERSONALES CONCURRENTES EN EL AGRESOR

\begin{tabular}{|l|c|c|c|c|}
\hline & $\begin{array}{c}\text { Consumo de } \\
\text { Alcohol }\end{array}$ & $\begin{array}{c}\text { Consumo de } \\
\text { Otro tipo de } \\
\text { Drogas }\end{array}$ & $\begin{array}{c}\text { Trastorno mental/ } \\
\text { Problemas } \\
\text { Psicológicos }\end{array}$ & \\
\hline $\begin{array}{l}\text { 1.-ASCENDIENTES u } \\
\text { OTROS }\end{array}$ & $\begin{array}{c}90 \\
11,7 \%\end{array}$ & $\begin{array}{c}103 \\
13,4 \%\end{array}$ & $\begin{array}{c}106 \\
14,1 \%\end{array}$ & $\begin{array}{c}769 \\
100 \%\end{array}$ \\
\hline 2.- MENORES & 28 & 11 & 9 & 197 \\
DESCENDIENTES & $14,2 \%$ & $5,6 \%$ & $4,7 \%$ & $100 \%$ \\
\hline 3.- VIOLENCIA & 568 & 167 & 140 & 3670 \\
EN LA PAREJA & $15,5 \%$ & $4,5 \%$ & $3,8 \%$ & $100 \%$ \\
\hline TOTAL & 686 & 281 & 255 & \\
\hline
\end{tabular}

Otras circunstancias personales concurrentes en el agresor, recogidas en la Tabla 2, son por su posible importancia merecedoras de consideración como factor psicosocial influyente: nos referimos a la situación de desempleo y la existencia de precariedad económica. Se ha comprobado que según los grupos de familiar agredido se hallaban en desempleo un 2-3\% de los agresores. Sin embargo, solamente el 1-2\% manifestaron necesidades económicas.

Tabla 2. Circunstancias personales concurrentes en el agresor, según tipo de violencia.

\begin{tabular}{|l|r|r|}
\hline 1) ASCENDIENTES & & \\
Consumo de alcohol & 103 & $11,7 \%$ \\
Consumo de otro tipo de drogas & 24 & $13,4 \%$ \\
Desempleo & 12 & $3 \%$ \\
Necesidades económicas & 106 & $2 \%$ \\
Trastorno Mental/Problema Psicolg. & & $14,1 \%$ \\
\hline 2) Menores descendientes & 28 & $14,2 \%$ \\
Consumo de alcohol & 11 & $5,6 \%$ \\
Consumo de otro tipo de drogas & 3 & $2 \%$ \\
Desempleo & 5 & $3 \%$ \\
Necesidades económicas & 9 & $4,7 \%$ \\
Trastorno mental /Problema Psicolg. & 568 & $15,5 \%$ \\
\hline 3) Mujer u hombre & 167 & $4,5 \%$ \\
Consumo de alcohol & 89 & $2 \%$ \\
Consumo de otro tipo de drogas & 30 & $1 \%$ \\
Desempleo & 140 & $3,8 \%$ \\
Necesidades económicas & & \\
Trastorno mental/ Problema Psicolg. & & \\
\hline
\end{tabular}




\section{INFORMES}

\section{Discusión}

La muestra, con ser importante, apenas alcanza a una pequeña parte de las sentencias sobre violencia doméstica. El estudio realizado puede considerarse representativo, pero sólo con estudios tendentes a contemplar la totalidad de los procedimientos sobre el objeto de estudio planteado se tendrá un conocimiento cabal del problema.

Aun siendo pequeña la relación entre violencia familiar y trastorno en la salud mental, sí se ha descrito como algunos trastornos delirantes, el consumo excesivo de alcohol y otras drogas, los comportamientos celotípicos, el trastorno antisocial, etc, pueden activar conductas violentas en personas impulsivas, siendo de mayor riesgo para quienes conviven con ellas. En un estudio con una muestra de 150 expedientes judiciales de malos tratos tramitados en los juzgados de distrito de Madrid (13), en un 22\% de los casos el alcoholismo había sido causa inmediata que provocó los malos tratos, y en menor medida otros motivos como el desempleo, los celos, motivos económicos o perturbación mental. En otro estudio de la Comunidad Valenciana (14), un 30\% de los hombres maltratadores eran consumidores habituales de alcohol y un 3\% de drogas. El Instituto Vasco de la Mujer, Emakunde, realizó en 1991 un estudio (15) sobre la mujer, y en él se elevaba la incidencia de consumo de alcohol por los agresores hasta alcanzar el $50 \%$ de la muestra analizada.

La violencia conyugal puede ser parcialmente efecto de un trastorno psicopatológico, habiéndose comprobado en nuestro medio la existencia de porcentajes superiores de historia psiquiátrica en personas agresoras que las tasas de prevalencia en la población general (7). Y también es causa de psicopatología en quienes padecen la agresión. Pero es adecuado plantearse límites a la explicación causal de las alteraciones psicológicas. Si no ¿cómo explicar que esta violencia se desarrolle fundamentalmente en el marco del hogar y en gran medida contra la esposa o compañera (16), manteniendo los agresores conductas adecuadas en otros ámbitos sociales?

La precariedad económica también se antoja factor presente en las situaciones de violencia familiar. En un estudio de Emakunde en 1997 (17), entre las mujeres víctimas de maltrato doméstico, atendidas en el programa de atención psicológica en Bizkaia y Alava durante los años precedentes, en cuanto al nivel socioeconómico de las encuestadas, el $34,7 \%$ eran de nivel bajo y otro $21 \%$ medio bajo.

Se han tomado algunas medidas institucionales frente a este tipo de agresiones $(6,19)$ pero teniendo en cuenta estos datos, entendemos que toda medida enfocada tanto a la atención como a la prevención de la violencia doméstica ha de ir orientada a favorecer la autonomía, dignidad y protección de las mujeres y los menores, con acciones no sólo orientadas a penalizar a los hombres que han agredido a las mujeres, sino hacia la consecución de espacios donde las relaciones estén dominadas por valores de respeto, justicia y equidad tanto en lo público como en lo privado. 
Se requieren estrategias transversales que articulen medidas para combatir la violencia enraizada en las normas, comportamientos e instituciones que vinculan a las personas, siendo los varones el objeto principal por ser quienes ocasionan mayoritariamente los daños.

La violencia de género ha de ser condenada social y jurídicamente en todas sus expresiones y con toda la fuerza posible, siendo rechazada como vía para la resolución de conflictos entre las personas. Se ha de cuestionar y modificar las estructuras autoritarias y no igualitarias donde dominan los modelos y actuaciones de masculinidad tradicional, caminando hacia su transformación y redefinición. Es necesario generar dispositivos y actuaciones sociopolíticas, educativas y sanitarias, de carácter preventivo y sensibilizador hacia el conjunto de la población, hacia los varones jóvenes y hacia las propias mujeres. Medidas y políticas reeducativas y asistenciales hacia quienes actúan violentamente o son personas potencialmente agresoras, para favorecer su detección precoz o su responsabilización efectiva con mejor participación en las relaciones familiares. Identificando las diversas formas de violencia masculina y abriendo medidas alternativas al Sistema de Justicia Criminal que sean realmente responsabilizadoras, más alláá del mero retribucionismo penitenciario. Se requieren protocolos de actuación, medidas y recursos suficientes para actuar con efectividad. Es más, es necesario el compromiso y la implicación de varones del ámbito profesional o social en general, rompiendo actitudes corporativas, sobre todo cara a la sensibilización, la reconstrucción moral y a la transformación cultural frente a todo tipo de violencia de género.

\section{NOTAS:}

${ }^{1}$ También en el Artículo 1 de la "Declaración sobre la eliminación de la violencia contra la mujer" de la ONU. Resolución 48/104, de 20 de diciembre de 1993.

${ }^{2}$ Ver datos del Instituto de la Mujer en http://www.mtas.es Por otro lado, según datos aportados por Emakunde, de las 42 muertes producidas en 2001, en la CAPV se han producido 2.

${ }^{3}$ En 1998 se parobó el primer Plan de Acción contra la Violencia Doméstica desde el Ministerio del Interior. En ese año, 35 mujeres fueron asesinadas por su pareja o expareja. En 1999, fueron 42 las mujeres asesinadas.

${ }^{4}$ Europa Press, 14-6-2001, recogiendo información del Congreso de la Sociedad Española de Medicina de Emergencias y Urgencias celebrado en Cádiz. 


\section{INFORMES}

\section{BIBLIOGRAFÍA}

1.- Carmona, L1 y Jiménez, ML. Malos tratos en mujeres. JANO, 2000, LVIII, 1335: 1075-1077.

2.- OMS. Programa normal sobre pruebas cientificas para las Políticas de salud; dando prioridad a las mujeres,. Recomendaciones éticas y de seguridad para la investigación sobre la violencia doméstica contra las mujeres. Ginebra, 1994.

3.- Clare, A. Hombres. La masculinidad en crisis. Taurus. Madrid, 2002.

4.- Bonino, L. "Violencia de género y prevención. El problema de la violencia masculina". En Actuaciones sociopolíticas preventivas de la violencia de género. UNAF, Madrid, 2000.

5.- Ferrer, E. "Violencia contra las mujeres: movilización general". JANO, 2002, Vol. LXI $n^{\circ}$ 1.392: 93-94.

6.- http://www.mtas.es, sitio web del Ministerio de Trabajo y Asuntos Sociales, con vínculo al Instituto de la Mujer.

7.- Echeburúa, E. y de Corral, P. Manual de violencia familiar. Siglo XXI Editorial, 1998.

8.- Amor Andrés, PJ. Variables sociodemográficas y psicopatológicas en mujeres víctimas de maltrato. Tesis Doctoral, UPV-EHU. Donostia, 2000.

9.- Villavicencio, P. "Mujeres maltratadas: Conceptualización y evaluación”. Clínica y Salud, 1993, Vol 4, n³: 215-228.

10.- Rey, T. Malos tratos domésticos. Barneko Albistaria, 2001, 87: 2-4.

11.- Larrion, JL y De Paul, J. "Mujer, violencia y salud". Med. Clin. (Barc), 2000, 115: 620-624.

12.- Rodríguez Vega, B; Fernández Liria, A. "La consideración psiquiátrica del maltrato a la mujer". Archivos de Psiquiatría, 2002, 65 (1): 1-3.

13.- Gutierrez, P. La administración de Justicia ante el problema de malos tratos a mujeres en el ámbito doméstico. Madrid, Documento R 3685/ 3166 del Centro de Documentación del Instituto de la Mujer. Madrid, 1989.

14.- Generalidad Valenciana, Consejería de Trabajo y Seguridad Social (1990). Incidencia de los malos tratos a mujeres en la Comunidad Valenciana. Serveis Socials, Estudis, 2001, 17. Valencia.

15.- Emakunde (Instituto Vasco de la Mujer). Informe sobre la situación de las mujeres en Euskadi. Vitoria-Gasteiz, 1991.

16.- Corsi, J. Violencia masculina en la pareja. Una aproximación al diagnóstico y a modelos de intervención. Ed. Paidós. Buenos Aires, 1995.

17.- Emakunde (Instituto Vasco de la Mujer). Mujer y maltrato doméstico en Bizkaia y Alava., 1997.

18.- Villavicencio, P. y Sebastián, J. Violencia doméstica: su impacto en la salud física y mental de las mujeres. Edita: Instituto de la Mujer, Ministerio de Trabajo y Asuntos Sociales. Madrid, 1999.

19.- Emakunde (Instituto Vasco de la Mujer). Protocolo interinstitucional contra el maltrato y agresiones sexuales a las mujeres. Emakunde, 2001, 43: 20-25.

(*) Ekimen, Investigación sobre Salud mental y Sustancias. www.ekimen.org

Correspondencia: Iñaki Markez, Médico Psiquiatra

Apdo Correos 276, 48993-Algorta. Bizkaia - Tfno: 94.4674979

imarkez@euskalnet.net

Recibido: 12-08-02 